\title{
Comparative Studies on the Interaction of Cochinchinenin A and Loureirin B with Bovine Serum Albumin
}

\author{
Tianming Yang, ${ }^{1}$ Hao Zhang, ${ }^{1}$ Haiyan Fu, ${ }^{1}$ Yuanbin She, \\ Can Huang, ${ }^{1}$ Yan Hu, ${ }^{1}$ and Liping Wang ${ }^{1}$ \\ ${ }^{1}$ Third Class Laboratory about Ethno-Medicine of State Administration of Traditional Chinese Medicine, College of Pharmacy, \\ South-Central University for Nationalities, Wuhan 430074, China \\ ${ }^{2}$ Key Laboratory of Catalysis and Materials Science of the State Ethnic Affairs Commission \& Ministry of Education, \\ College of Chemistry and Materials Science, South-Central University for Nationalities, Wuhan 430074, China
}

Correspondence should be addressed to Haiyan Fu; fuhaiyan76@yahoo.com.cn and Yuanbin She; sheyb@bjut.edu.cn

Received 9 March 2013; Accepted 7 May 2013

Academic Editor: Renata Diniz

Copyright (c) 2013 Tianming Yang et al. This is an open access article distributed under the Creative Commons Attribution License, which permits unrestricted use, distribution, and reproduction in any medium, provided the original work is properly cited.

\begin{abstract}
This paper describes the simple, sensitive, and effective spectrophotometric methods based on ultraviolet, fluorescence and circular dichroism for revealing the interactional mechanism of Cochinchinenin A (CA) and Loureirin B (LB) with bovine serum albumin (BSA). Under simulated physiological conditions, it was demonstrated that the fluorescence quenching mechanisms between CA (or LB) and BSA as a static quenching mode, or a combined quenching (dynamic and static quenching) mode were related to concentration level of CA (or LB). The binding distance $\left(r_{\mathrm{CA}}, r_{\mathrm{LB}}\right)$ and the quenching efficiency $\left(K_{\mathrm{SV}}\right)$, especially for the binding constants value of ligands to BSA, were affected by the methoxyl group at position 4 at different temperatures. The corresponding thermodynamic parameters were also obtained and indicated that electrostatic forces play a major role in the formation of the LB-BSA complex, but probably a combined force for CA-BSA complex. Furthermore, synchronous fluorescence spectroscopy and circular dichroism spectra demonstrated that the secondary structures of BSA were changed to varying degrees by the binding of CA (or LB).
\end{abstract}

\section{Introduction}

Cochinchinenin A (CA) and Loureirin B (LB) are important bioactive ingredients of Dragon's blood, which is a deep red resin and has been used as a famous traditional medicine since ancient times by many cultures. The deep red resin of Dracaena cochinchinensis (Lour.) S. C. Chen (Agavaceae), believed to be the original source of Chinese dragon's blood, has been widely used in traditional Chinese medicine for promoting blood circulation, treating trauma, relieving pain, visceral hemorrhages, antimicrobial, mainly for the treatment of coronary heart disease, cerebral infarction, and other thrombotic diseases [1-4]. CA (4'-hydroxy2,6-dimethoxydihydrochalcone) and LB (4'-hydroxy-2,4,6trimethoxydihydrochalcone) (Figure 1) as the synergistic bioactive ingredients of Dragon's Blood extracts were shown to have certain antithrombotic effects, antiplatelet aggregation effects, anti-inflammatory, and analgesic effects [5-8].
In spite of these broad pharmacological actions of CA and LB mentioned earlier, their effects on proteins and the mechanisms of actions are poorly understood. Additionally, the interaction between drugs and proteins could significantly affect the transportation and distribution of $\mathrm{CA}$ and $\mathrm{LB}$ and also affect their therapeutic activity and toxicity $[9,10]$. As seen in Figure 1, the only difference in structure is that there is one more methoxyl group in 4-substitution of LB. Consequently, studies on the protein-drug binding are clearly important for the explanation of the reaction mechanisms, providing a pathway to understand the pharmacokinetics and pharmacodynamic mechanisms of CA and LB in vivo.

Among all proteins, serum albumin is the most abundant protein constituent in blood plasma and plays important roles in the disposition and transport of various exogenous and endogenous ligands to particular biological targets. The effectiveness of a few compounds as pharmaceutical agents depends on their binding ability to serum albumin [11-13]. 


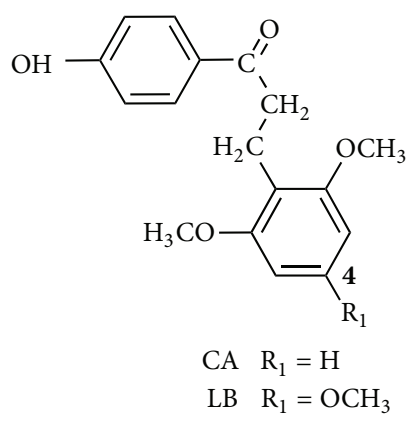

FIGURE 1: Molecular structures of Cochinchinenin A and Loureirin B.

In recent years, the binding properties of serum albumin and drugs were investigated by many researchers and human serum albumin (HSA) has been usually used as a model protein in evaluating protein-drug interactions [14-16]. On the other hand, bovine serum albumin (BSA) and HSA exhibit similar binding chemistry due to the high percentage of sequence identities between the two proteins. So BSA has been commonly used because of its ready availability and affordability [17-19].

In the present study, the fluorescence spectroscopy, ultraviolet spectroscopy, and circular dichroism (CD) techniques were employed to explore the influence of methoxyl group on the interactions of CA or LB with BSA under simulative physiological conditions [20]. These researching results not only reveal the mechanism of interaction of CA (or LB) and BSA, but also help to discover the transportation and distribution of the Dragon's blood drugs in vivo and explain the toxicity at the molecular level. Moreover, this study may provide some available information for drug design and pharmaceutical research.

\section{Experimental}

2.1. Apparatus. All fluorescence spectra were measured on LS-55 (PerkinElmer, USA) spectrofluorometer equipped with a thermostatic bath (PerkinElmer, USA), a xenon lamp, and a $1.0 \mathrm{~cm}$ quartz cell. UV-vis absorption spectra were measured on a LAMBDA-35 (PerkinElmer, USA) spectrophotometer with the use of a $1.0 \mathrm{~cm}$ quartz cell. CD spectra were measured on a J-810 spectropolarimeter (Jasco, Tokyo, Japan) with a $0.1 \mathrm{~cm}$ quartz cell under constant nitrogen flush. All pH measurements were made with a $\mathrm{pHs}-3 \mathrm{C}$ digital $\mathrm{pH}$ meter (Jinmai, Shanghai, China). All experiments, unless specified otherwise, were carried out at room temperature.

2.2. Reagents. BSA was obtained from Sigma (USA) and was dissolved in a Tris/ $\mathrm{HCl}\left(0.05 \mathrm{~mol} \mathrm{~L}^{-1}, \mathrm{pH} 7.40\right)$ buffer to form a BSA solution with a concentration of $2.0 \times$ $10^{-6} \mathrm{~mol} \mathrm{~L}^{-1}$ and was kept in dark at $277 \mathrm{~K}\left(4^{\circ} \mathrm{C}\right)$. Tris $/ \mathrm{HCl}$ buffer $\left(0.05 \mathrm{~mol} \mathrm{~L}^{-1}, \mathrm{pH} 7.40\right)$ containing $0.10 \mathrm{~mol} \mathrm{~L}^{-1} \mathrm{NaCl}$ was selected to keep the $\mathrm{pH}$ constant and to maintain the ionic strength of the solution. CA and LB were provided by Professor Wenjie Lu (Guangxi Institute of Traditional
Medicine and Pharmaceutical Sciences) and were prepared in ethanol as stock solution $\left(1.0 \times 10^{-4} \mathrm{~mol} \mathrm{~L}^{-1}\right)$. All other reagents were of analytical grade and all the water used in the experiments was double-distilled.

\subsection{Procedures}

2.3.1. Fluorescence Spectroscopy. A working solution containing $1.0 \mathrm{~mL}$ BSA stock solution was titrated manually by successive additions of $1.0 \mathrm{~mL}$ CA (or LB) stock solution and Tris/HCl buffer with micropipette, and then the mixture was diluted to the standard volumetric flask with water and incubated for $20 \mathrm{~min}$ to equilibrate the system. Fluorescence quenching measurements were carried out by keeping the concentration of BSA fixed at $2.0 \times 10^{-6} \mathrm{~mol} \mathrm{~L}^{-1}$ and that of CA (or LB) varied from 0 to $1.8 \times 10^{-5} \mathrm{~mol} \mathrm{~L}^{-1}$. The fluorescence emission spectra were measured at 288, 298, and $310 \mathrm{~K}$; the width of the excitation and emission slits was set at 6.0 and $10.0 \mathrm{~nm}$, respectively. An excitation wavelength of $282 \mathrm{~nm}$ was chosen and the emission wavelength was recorded from 300 to $450 \mathrm{~nm}$ while the scanning rate was $1500 \mathrm{~nm} / \mathrm{min}$. On the other hand, the excitation and emission slits were also set at 6.0 and $10.0 \mathrm{~nm}$ for the synchronous fluorescence spectra measurements, respectively. Blanks corresponding to the buffer were subtracted to correct background fluorescence. And the $\Delta \lambda=60 \mathrm{~nm}$, excitation wavelength was set from 200 to $350 \mathrm{~nm}$; experiments were carried out at the temperature $310 \mathrm{~K}$.

2.3.2. UV-Vis Absorbance and CD Spectroscopy. The UV-vis absorption spectra of CA (or LB) solution with the concentration of $2.0 \times 10^{-6} \mathrm{~mol} \mathrm{~L}^{-1}$ were measured in the range of $300-450 \mathrm{~nm}$ at $310 \mathrm{~K}$. CD measurements were performed at $0.2 \mathrm{~nm}$ intervals over a wavelength range of 190-280 $\mathrm{nm}$ and the scanning speed was set at $200 \mathrm{~nm} / \mathrm{min}$. The concentration of BSA was kept at $2.0 \times 10^{-6} \mathrm{~mol} \mathrm{~L}^{-1}$ and the molar ratio of BSA to AC (LB) was varied as $1: 0,1: 5$, and $1: 10$.

\section{Results and Discussion}

3.1. Fluorescence Quenching of BSA by CA or LB. Proteins are considered to have intrinsic fluorescence due to the presence of amino acid residues, mainly two tryptophan (Trp) residues, Trp-212 and Trp-134 for BSA. Similar to HSA, Trp-212 is located in subdomain IIA whereas Trp-134 is localized in the subdomain IA [21]. Fluorescence quenching is the decrease of the quantum yield of fluorescence from a fluorophore induced by a variety of molecular interactions such as excited-state reactions, molecular rearrangements, energy transfer, ground-state complex formation, and collision quenching $[22,23]$. Different mechanisms of fluorescence quenching are usually classified as dynamic quenching and static quenching, which are caused by diffusion and ground-state complex formation, respectively. And they have different dependence on temperature; it is known that higher temperatures result in larger diffusion coefficients, and the dynamic quenching constants are expected to increase when 


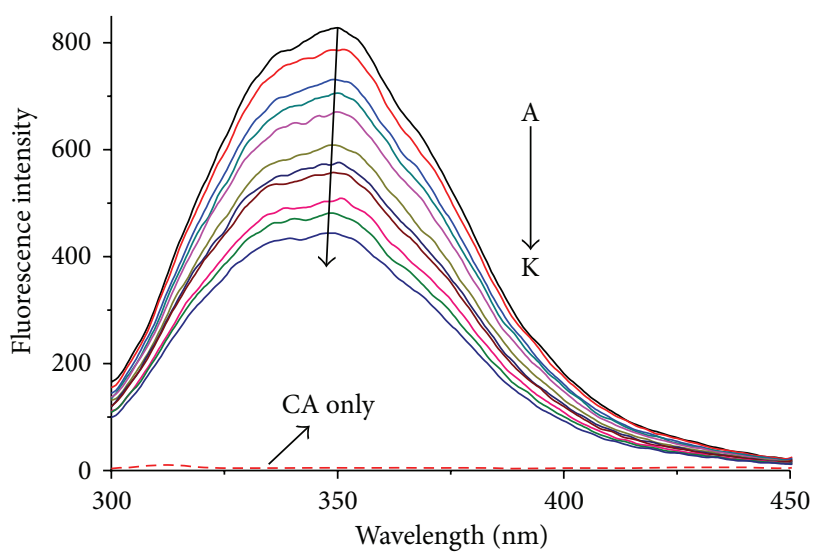

(a)

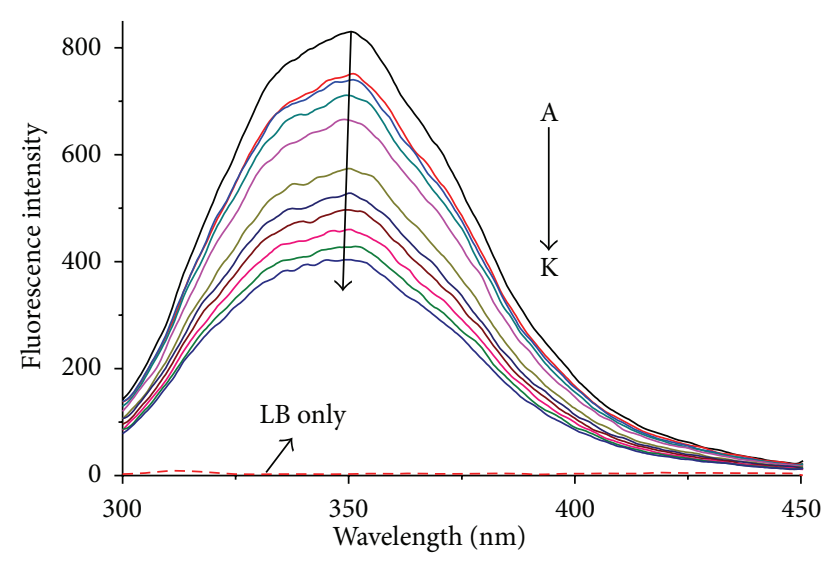

(b)

FIGURE 2: Emission spectra of BSA in the presence of various concentrations of CA (a) and LB (b) $\left(T=310 K, \lambda_{\mathrm{ex}}=282 \mathrm{~nm}, \mathrm{pH}=7.40\right)$. $\mathrm{C}(\mathrm{BSA})=2 \times 10^{-6} \mathrm{~mol} \mathrm{~L}^{-1} ; \mathrm{C}(\mathrm{CA}$ or LB $) /\left(10^{-6} \mathrm{~mol} \mathrm{~L}^{-1}\right), \mathrm{A}-\mathrm{K}: 0,0.8,2.5,3.5,5.0,8.0,10.0,12.0,14.0,16.0,18$.

there is a rise in temperature. In contrast, increased temperature is likely to result in decreased stability of complexes and thus lower values of the static quenching constants. Dynamic and static quenching can be distinguished by the emission spectra before and after two samples interact as well [24].

In this work, the fluorescence quenching spectra of BSA in the presence of different concentrations of CA (or LB) were measured to elucidate the quenching mechanism in Figure 2. As shown in Figure 2, BSA exhibited strong fluorescence emission at about $350 \mathrm{~nm}$ on excitation at $282 \mathrm{~nm}$, while CA and LB showed very weak intrinsic fluorescence under the same experimental conditions. Furthermore, when BSA was added with increasing amounts of CA (or LB), a remarkable intrinsic fluorescence decrease of BSA was observed as well as the slight blue shift of the maximum emission wavelength. This suggested that the intrinsic fluorescence of the tryptophan residues was quenched and the microenvironment around BSA was changed after the addition of CA or LB.

Fluorescence quenching is described by the well-known Stern-Volmer equation which can also classify the type of fluorescence quenching process as static or dynamic [25]. Consider

$$
\frac{F_{0}}{F}=1+K_{\mathrm{SV}}[Q]=K_{q} \tau_{0}[Q]
$$

where $F_{0}$ and $F$ are the steady-state fluorescence intensities of the protein in the absence and presence of the quencher (CA or LB), respectively; $K_{\mathrm{SV}}$ is the Stern-Volmer quenching constant; $K_{q}$ is the bimolecular quenching rate constant; $\tau_{0}$ is the average lifetime of the molecule without the quencher and its value is about $10^{-8} \mathrm{~s}[26] ;[Q]$ is the concentration of the quencher. $K_{\mathrm{SV}}$ was acquired from the slope of linear regression of a plot of $F_{0} / F$ versus $[Q]$. In many cases, the fluorophore can be quenched by both mechanisms with the same quencher, while the Stern-Volmer plot exhibits an upward curvature for the distinct situation, concave toward the $y$-axis at high [Q] $[27,28]$. Accordingly, the $F_{0} / F$ is related to $[Q]$ by the following modified Stern-Volmer equation:

$$
\begin{aligned}
\frac{F_{0}}{F} & =\left(1+K_{D}[Q]\right)\left(1+K_{S}[Q]\right) \\
& =1+\left(K_{D}+K_{S}\right)[Q]+K_{D} K_{S}[Q]^{2},
\end{aligned}
$$

where $K_{S}$ and $K_{D}$ are the static and dynamic quenching constants, respectively. It is second order in $[Q]$ and thus leads to upward curve plots of $F_{0} / F$ versus [Q] at higher $[Q]$ arising from a combined quenching (both dynamic and static) process.

Figure 3 displays the Stern-Volmer plots of the quenching of BSA tryptophan residues fluorescence by CA or LB at different temperatures $(288,298,310 \mathrm{~K})$ and the corresponding results at different temperatures $(\mathrm{pH}=7.40)$ are shown in Table 1. As seen the little plots (insert in Figure 3) of $F_{0} / F$ for BSA versus the low concentrations of CA (or LB), ranging from $0.25 \times 10^{-5}$ to $1.4 \times 10^{-5} \mathrm{~mol} \mathrm{~L}^{-1}$, are linear and they exhibit a good linear relationship. However, the quenching constant $K_{\mathrm{SV}}$ in Table 1 decreased with the increasing temperature and constant $K_{q}$ (such as $4.056 \times$ $10^{12}$ and $4.415 \times 10^{12} \mathrm{Lmol}^{-1} \mathrm{~s}^{-1}, 310 \mathrm{~K}$ ) is much larger than $2.0 \times 10^{10} \mathrm{Lmol}^{-1} \mathrm{~s}^{-1}$, which is the value of the maximum diffusion collision quenching rate constant [29]. Therefore, the interaction between BSA and CA (or LB) at low concentrations is a static quenching process resulting from the formation of BSA-CA (BSA-LB) complex. For the quenching constants $K_{\mathrm{SV}}$ of $\mathrm{LB}$ are greater than that of CA at the identical external experimental conditions, so quenching efficiency of LB is more than that of CA. Lastly, the upward curves showed in Figure 2 further verify that the fluorescence quenching mechanism of CA and LB at high concentrations is a combined one.

3.2. Binding Constant and the Number of Binding Sites. It has been reported that there are independent binding sites in the bimolecular, and the binding constant $K_{A}$ as well as number 


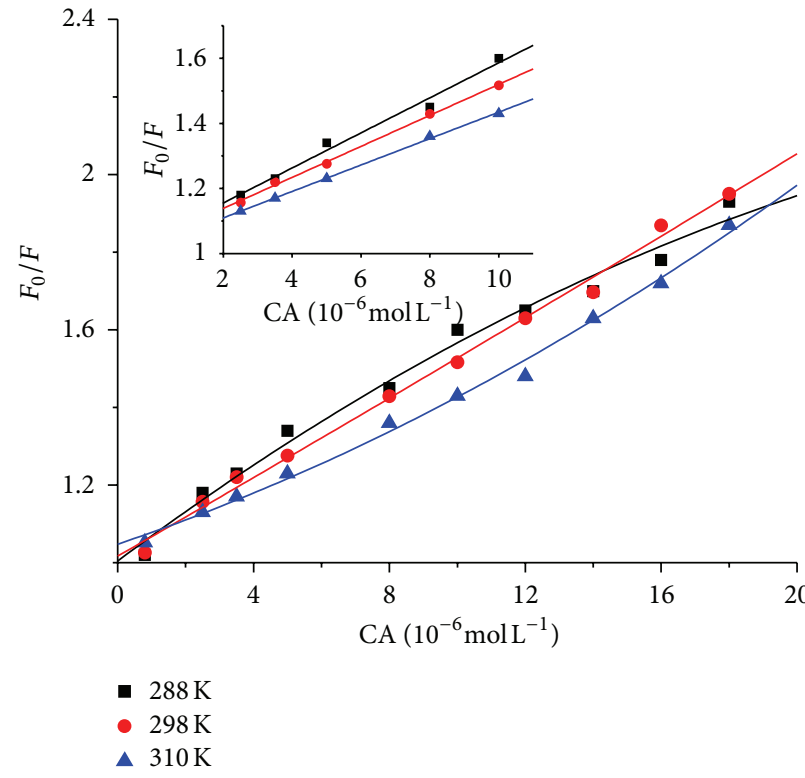

(a)

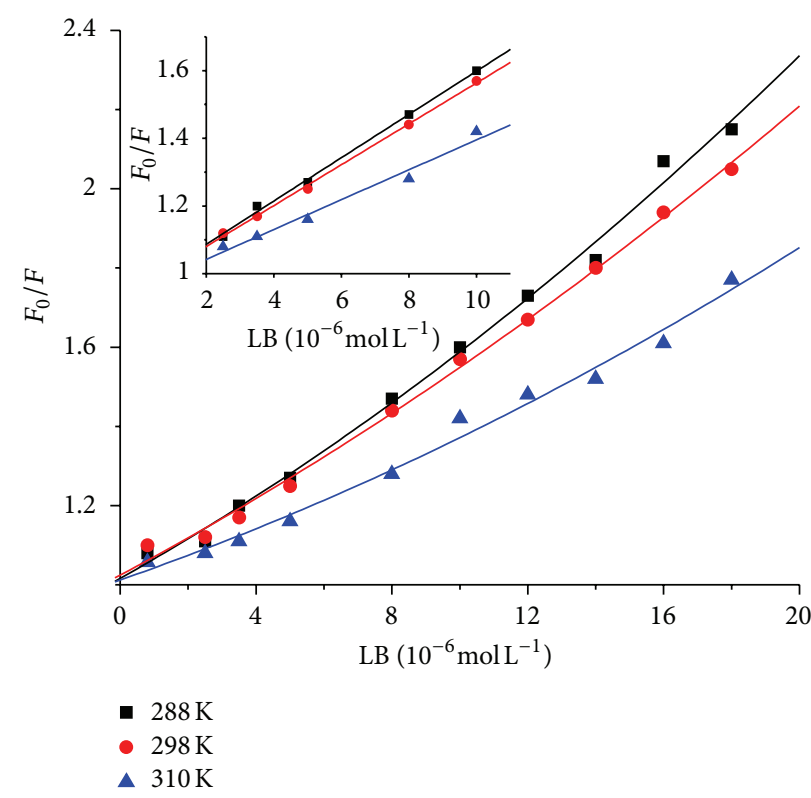

(b)

Figure 3: Stern-Volmer plots for the quenching of BSA by CA (a) and LB (b) at different temperatures $\left(\lambda_{\text {ex }}=282 \mathrm{~nm}, \mathrm{pH}=7.40\right), \mathrm{C}(\mathrm{BSA})=$ $2 \times 10^{-6} \mathrm{~mol} \mathrm{~L}^{-1}$.

TABLE 1: Stern-Volmer quenching constant $K_{\mathrm{SV}}$ of BSA by CA and LB at different temperatures.

\begin{tabular}{llccc}
\hline Compound & $\begin{array}{c}T \\
(\mathrm{~K})\end{array}$ & $\begin{array}{c}K_{\mathrm{SV}} \\
\left(\mathrm{Lmol}^{-1}\right)\end{array}$ & $\begin{array}{c}K_{q} \\
\left(\mathrm{Lmol}^{-1}\right)\end{array}$ & $R$ \\
\hline \multirow{3}{*}{ CA } & 288 & $5.382 \times 10^{4}$ & $5.382 \times 10^{12}$ & 0.9931 \\
& 298 & $4.759 \times 10^{4}$ & $4.759 \times 10^{12}$ & 0.9990 \\
& 310 & $4.056 \times 10^{4}$ & $4.056 \times 10^{12}$ & 0.9995 \\
\hline \multirow{2}{*}{ LB } & 288 & $6.399 \times 10^{4}$ & $6.399 \times 10^{12}$ & 0.9986 \\
& 298 & $6.043 \times 10^{4}$ & $6.043 \times 10^{12}$ & 0.9991 \\
& 310 & $4.415 \times 10^{4}$ & $4.415 \times 10^{12}$ & 0.9884 \\
\hline
\end{tabular}

$R$ is the correlation coefficient.

of binding site $n$ could be determined using the equation [30, 31]

$$
\log \frac{\Delta F}{F}=\log \frac{F_{0}-F}{F}=\log K_{A}+n \log [Q],
$$

where $\Delta F$ is the difference value of fluorescence in the absence $\left(F_{0}\right)$ and presence $(F)$ of the quenching compound at concentration $[Q] ; K_{A}$ is the binding constant of interaction between CA (or LB) and BSA; $n$ is the number of binding sites. The plots of $\log \left[\left(F_{0}-F\right) / F\right]$ versus $\log [Q]$ presented in Figure 4 at different temperatures are linear. Based on (3), the values of $K_{A}$ and $n$ can be calculated by the slope and intercept of the double-logarithmic regression curves in Figure 4 and the corresponding results were listed in Table 2.

In Table 2, the results suggest that the binding constants for the LB-BSA system decrease with the increasing temperatures and indicate that the capacity of LB binding to BSA was reduced for the increasing temperature leading to the increasing diffusion coefficient and the lower stability of the complex. Conversely, the binding constants for the CA-BSA systems do not decrease with the increasing temperature, which may be due to the influence of combined quenching (both dynamic and static) process. Furthermore, the $K_{A}$ (LBBSA) at different temperatures are much larger than $K_{A}$ (CABSA), which indicated that $L B$ has a much higher affinity than CA for binding to BSA. The larger $K_{A}$ implies the increased stability of the BSA-drug complex, which suggests that the addition of methoxyl group has effected on the interaction between LB and BSA. The binding numbers $(n)$ vary in the ranges from 0.837 to 0.863 and 1.139 to 1.152 for CA-BSA and LB-BSA, respectively. The values of $n$ are approximately equal to 1 , indicating that there is one binding site in BSA for both $\mathrm{CA}$ and LB during their interaction [31].

3.3. Thermodynamic Parameters and Nature of the Binding Forces. The interaction forces between small molecules and biological macromolecules may include hydrogen bonds, van der Waals forces, hydrophobic and electrostatic interactions, and so forth [32]. Generally, free energy change $\Delta G$ reflects the possibility of reaction, while enthalpy change $\Delta H$ and entropy change $\Delta S$ are the main quantities for judging the nature of the binding forces. Therefore, the thermodynamic parameters $(\Delta G, \Delta H$, and $\Delta S)$ of interaction are important to characterize the interaction force between CA (or LB) and BSA [33]. From the viewpoint of thermodynamics, $\Delta H>0$ and $\Delta S>0$ suggest hydrophobic forces; $\Delta H<0$ and $\Delta S<0$ imply van der Waals forces and hydrogen bonds; $\Delta H<0$ and $\Delta S>0$ reflect electrostatic forces. The thermodynamic 
TABLE 2: The binding constants and thermodynamic parameters of CA-BSA and LB-BSA systems at different temperatures.

\begin{tabular}{|c|c|c|c|c|c|c|c|}
\hline Compound & $\begin{array}{c}T \\
(\mathrm{~K})\end{array}$ & $\begin{array}{c}K_{A} \\
\left(\mathrm{Lmol}^{-1}\right)\end{array}$ & $n$ & $R$ & $\begin{array}{c}\Delta G \\
\left(\mathrm{kJmol}^{-1}\right)\end{array}$ & $\begin{array}{c}\Delta H \\
\left(\mathrm{kJmol}^{-1}\right)\end{array}$ & $\begin{array}{c}\Delta S \\
\left(\mathrm{Jmol}^{-1} \mathrm{k}^{-1}\right)\end{array}$ \\
\hline \multirow{3}{*}{$\mathrm{CA}$} & 288 & $8.905 \times 10^{3}$ & 0.837 & 0.9932 & -21.78 & & \\
\hline & 298 & $9.184 \times 10^{3}$ & 0.850 & 0.9979 & -22.61 & & \\
\hline & 310 & $8.992 \times 10^{3}$ & 0.863 & 0.9997 & -23.47 & & \\
\hline \multirow{3}{*}{ LB } & 288 & $3.278 \times 10^{5}$ & 1.139 & 0.9705 & -30.41 & \multirow{3}{*}{-13.54} & \multirow{3}{*}{58.749} \\
\hline & 298 & $2.866 \times 10^{5}$ & 1.140 & 0.9988 & -31.13 & & \\
\hline & 310 & $2.199 \times 10^{5}$ & 1.152 & 0.9950 & -31.71 & & \\
\hline
\end{tabular}

$R$ is the correlation coefficient.

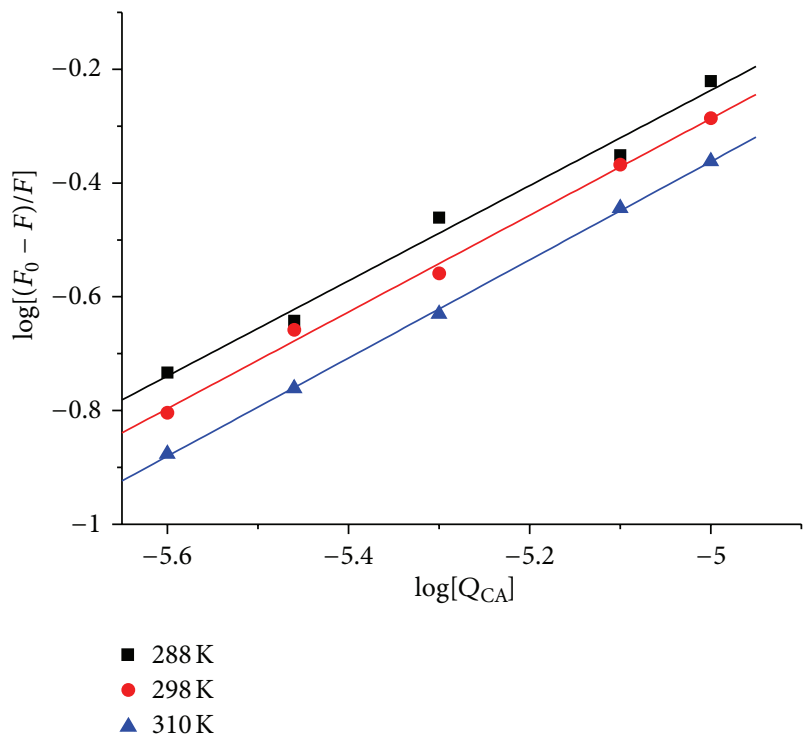

(a)

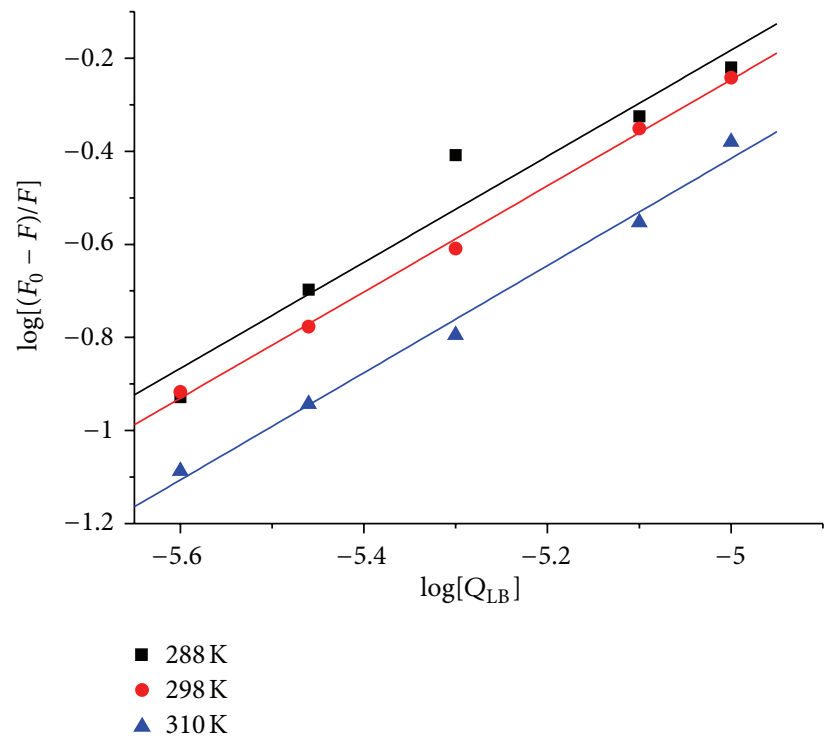

(b)

FIGURE 4: Plots of $\log \left[\left(F_{0}-F\right) / F\right]$ versus $\log \left[Q_{\mathrm{CA}}\right]$ and $\log \left[Q_{\mathrm{LB}}\right]$ at different temperatures.

parameters of BSA-LB complexes were calculated using the following Van't Hoff equation [34]:

$$
\ln K_{A}=-\frac{\Delta H}{R T}+\frac{\Delta S}{R} \text {. }
$$

If the temperature does not vary significantly, $\Delta H$ can be regarded as a constant. And the free energy changes $(\Delta G)$ at different temperatures can be calculated from the following equation:

$$
\Delta G=\Delta H-T \Delta S=-R T \ln K_{A},
$$

where $R$ is the gas constant and $K_{A}$ is the binding constant at the corresponding experimental temperature $T$. According to the binding constants $K_{A}$ of LB-BSA obtained at the three temperatures $(288,298$, and $310 \mathrm{~K})$, the values of $\Delta H$ and $\Delta S$ are obtained from the slope and intercept of the fitted curves of $\ln K_{A}$ versus $1 / T$, respectively. But to CA, the $K_{A}$ does not decrease with the increasing temperature $T$, and then the $\ln K_{A}$ and $1 / T$ of CA dissatisfies the Van't Hoff equation. As seen in Table 2, the values of thermodynamic parameters $(\Delta G, \Delta H$ and $\Delta S$ ) are obtained and presented.
From Table 2, it shows that the negative values of $\Delta G$ indicate that the binding processes are spontaneous for both CA-BSA and LB-BSA complex. The negative $\Delta H$ and positive $\Delta S$ values indicate that electrostatic forces play a major role in the formation of the LB-BSA complex $[32,34]$. But to CA, it did not meet one of the three situations of the viewpoint of thermodynamics, so we deduced that it is probably a combined force in the formation of the CA-BSA complex. Furthermore, the values of the thermodynamic parameters and the nature of the binding forces for these two systems are very different, which can be attributed to their different molecular structure and the different molecular weight.

3.4. Energy Transfer from BSA to $C A$ and $L B$. The purpose of study on the energy transfer in biochemistry is that the efficiency of transfer can be used to calculate the distance between the ligands and the tryptophan residues in the protein. According to Förster's nonradioactive energy transfer theory [35], the energy transfer will take place under the following conditions: first, the donor can produce fluorescence; second, fluorescence emission spectrum of 


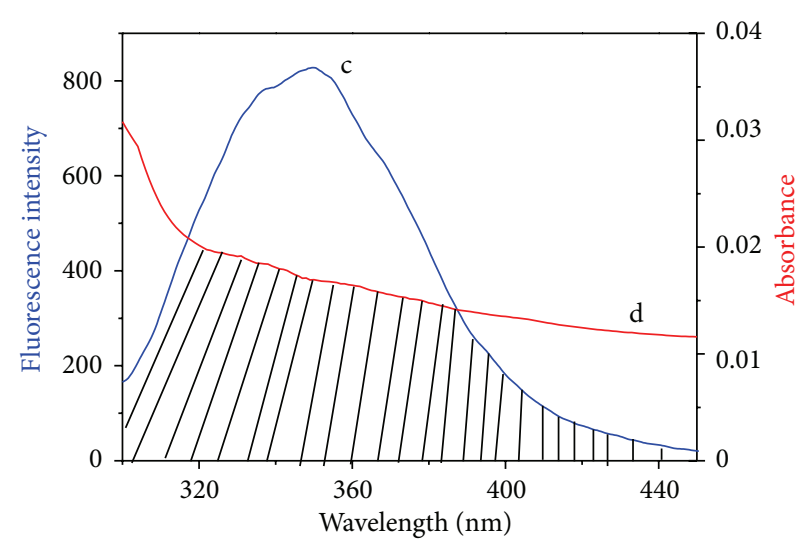

(a)

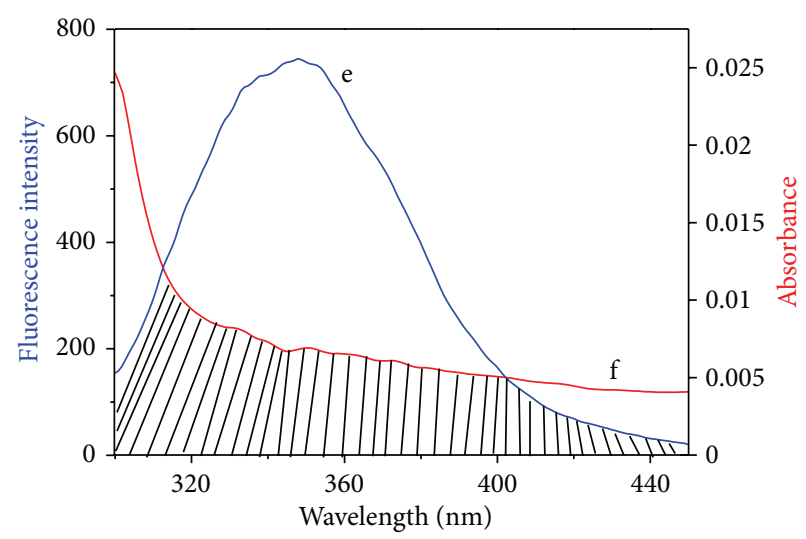

(b)

FIGURE 5: The overlap of fluorescence emission spectrum of BSA (c, e) and absorption spectrum of CA (d) and LB (f) $(T=310 \mathrm{~K}, \mathrm{pH}=7.40)$, $\mathrm{C}(\mathrm{BSA})=\mathrm{C}(\mathrm{CA})=\mathrm{C}(\mathrm{LB})=2.0 \times 10^{-6} \mathrm{~mol} \mathrm{~L}^{-1}$.

the donor and UV-vis absorbance spectrum of the acceptor must overlap significantly; and third, the distance between the donor and the acceptor is lower than $8 \mathrm{~nm}$. Based on this theory, the energy transfer efficiency $(E)$ is not only related to the distance $(r)$ between the donor and receptor, but also Förster's critical distance $\left(R_{0}\right)$. The values of $E$ are calculated using the equation

$$
E=1-\frac{F}{F_{0}}=\frac{R_{0}^{6}}{R_{0}^{6}+r^{6}},
$$

where $F$ and $F_{0}$ are the same as previously mentioned and $R_{0}$ is the critical distance when the energy transfer efficiency is $50 \%$. The value of $R_{0}$ was calculated using the equation

$$
R_{0}^{6}=8.79 \times 10^{-25} K^{2} n^{-4} \phi J,
$$

where $K^{2}$ is the spatial orientation factor of the dipole, $n$ is the refractive index of medium, $\varphi$ is fluorescence quantum yield of the donor, and $J$ is overlap integral of the fluorescence emission spectrum of donor and absorption spectrum of the acceptor (Figure 5). And $J$ can be calculated by the following equation:

$$
J=\frac{\sum F(\lambda) \varepsilon(\lambda) \lambda^{4} \Delta \lambda}{\sum F(\lambda) \Delta \lambda},
$$

where $F(\lambda)$ is the fluorescence intensity of the donor at wavelength $\lambda$, and $\varepsilon(\lambda)$ is the molar absorption coefficient of the acceptor at wavelength $\lambda$. According to (6)-(8), J, $R_{0}$, and $E$ can be obtained; so the value of $r$ also can be calculated.

Figure 6 is the overlap of the fluorescence spectra of BSA and the UV absorption spectra of CA or LB when the molar ratio is $1: 1$ and at $310 \mathrm{~K}$. The values of $J$ are obtained by integrating the overlap in the spectra (Figure 4), $1.336 \times$ $10^{-14}$ and $5.524 \times 10^{-15} \mathrm{~cm}^{3} \mathrm{Lmol}^{-1}$ for CA-BSA and LB-BSA, respectively. In the present case, $K^{2}=2 / 3, n=1.36$, and $\varphi=$ $0.15[36,37]$. Combined with (6)-(8), we can calculate that $E=0.05, R_{0}=1.23 \mathrm{~nm}$, and $r_{\mathrm{CA}}=2.01 \mathrm{~nm}(310 \mathrm{~K})$ for CABSA system and $E=0.05, R_{0}^{\prime}=1.06 \mathrm{~nm}$ and $r_{\mathrm{LB}}=1.73 \mathrm{~nm}$
(310 K) for LB-BSA system. Hence, the donor to acceptor distance $(r)$ in either system is less than $7 \mathrm{~nm}$ and $0.5 R_{0}<r<$ $1.5 R_{0}$, which indicates the energy transfer from BSA to CA or LB occurred with high possibility [38]. In accordance with Förster's nonradiative energy transfer theory, it is proved once again that the static quenching was presence in the interaction between CA (or LB) and BSA. And the donor to acceptor distance $(r)$ of LB is closer than that of CA, which indicates that the addition of methoxyl group is helpful for LB binding to BSA.

\subsection{Effect of $C A$ and $L B$ on Conformational Changes of BSA}

3.5.1. Synchronous Fluorescence Spectroscopy Studies. Synchronous fluorescence spectroscopy is a useful method to provide some information about the changes of molecular microenvironment and the environment of amino acid residues [39]. This is carried out by measuring the shift of maximum emission wavelength $\left(\lambda_{\max }\right)$, which corresponds to the changes of polarity around the chromophore molecule. When the wavelength shift $(\Delta \lambda)$ between excitation and emission wavelength set at $60 \mathrm{~nm}$, the characteristic information of tryptophan residues in protein was given [40]. And the synchronous fluorescence spectra of BSA upon addition of $\mathrm{CA}$ or LB at $310 \mathrm{~K}$ are shown in Figure 7. It shows that the fluorescence intensity of tryptophan decreased regularly with increasing $\mathrm{CA}$ (or LB) concentration, with the maximum emission wavelength removed from 278 to $280 \mathrm{~nm}$ for CA and from 279 to $281 \mathrm{~nm}$ for LB. These two shifts indicate that the conformations of the BSA proteins have been changed on the addition of CA or LB. Furthermore, the red-shift effects demonstrate that the polarity around the tryptophan residues increased and the hydrophobicity decreased with CA and LB.

3.5.2. Circular Dichroism Studies. In order to further investigate the conformational changes, the $\mathrm{CD}$ which is sensitive to variation of the structure of biological macromolecules was used to monitor the secondary structural change of protein upon interaction with CA and LB. And the CD spectra of 


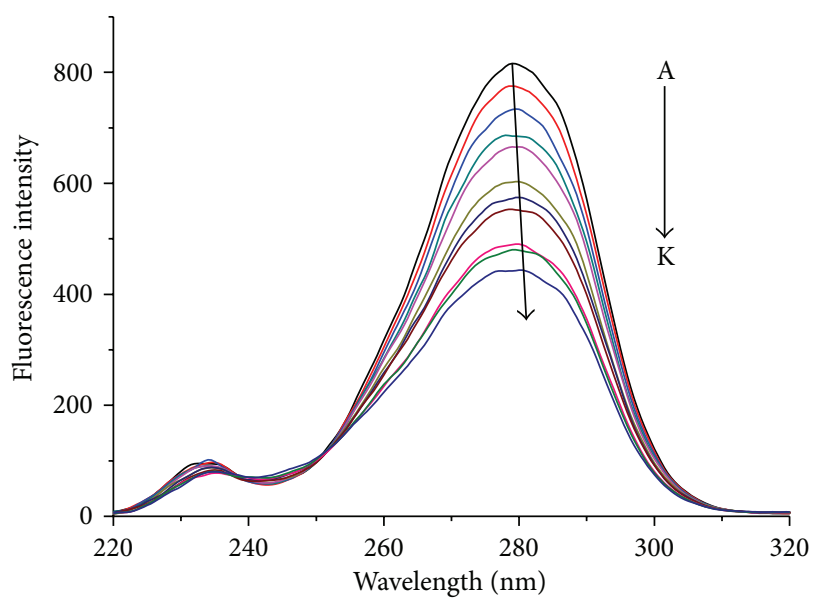

(a)

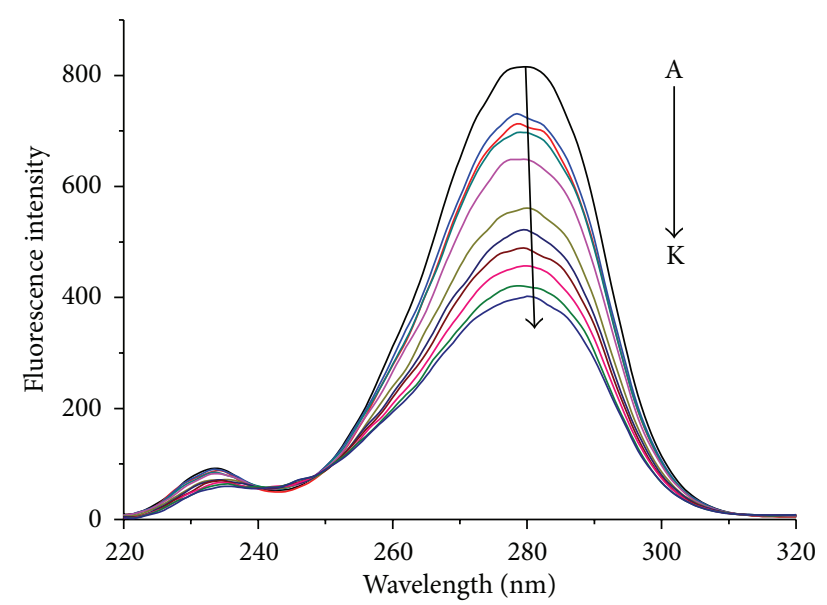

(b)

FIGURE 6: Synchronous fluorescence spectrum of BSA in the presence of CA (a) and LB (b) $(T=310 \mathrm{~K}, \Delta \lambda=60 \mathrm{~nm}, \mathrm{pH}=7.40)$. C(BSA) $=2 \times 10^{-6} \mathrm{~mol} \mathrm{~L}^{-1} ; \mathrm{C}(\mathrm{CA}) /\left(10^{-6} \mathrm{~mol} \mathrm{~L}^{-1}\right), \mathrm{A}-\mathrm{K}: 0,0.8,2.5,3.5,5.0,8.0,10.0,12.0,14.0,16.0,18.0$.

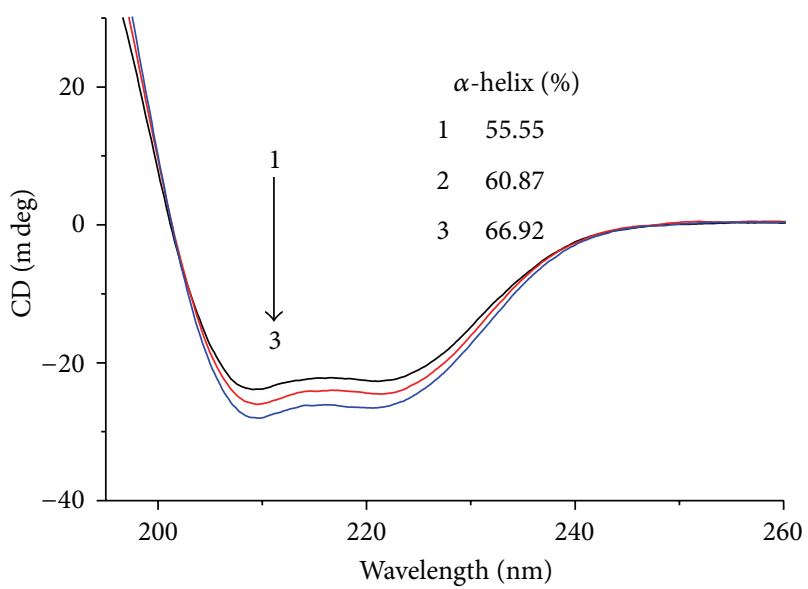

(a)

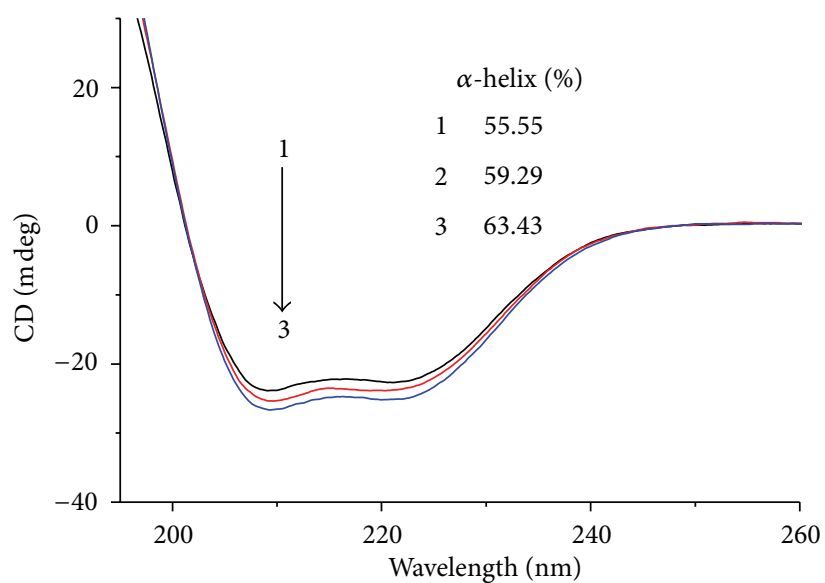

(b)

Figure 7: CD spectra of BSA in the presence of CA (a) and LB (b) $(T=310 \mathrm{~K}, \mathrm{pH}=7.40)$. C(BSA $)=2.0 \times 10^{-6} \mathrm{~mol} \mathrm{~L}^{-1} ; \mathrm{C}(\mathrm{CA}$ or LB $) /\left(10^{-6}\right.$ $\left.\mathrm{mol} \mathrm{L} \mathrm{L}^{-1}\right), 1-3: 0,10,20$.

BSA in the range of $185 \mathrm{~nm}$ to $260 \mathrm{~nm}$ were measured in the absence and addition of CA or LB, as shown in Figure 7. The $\mathrm{CD}$ results are expressed in terms of mean residue ellipticity (MRE) in degree $\mathrm{cm}^{2} \mathrm{dmol}^{-1}$ that can be calculated by following equation [41]:

$$
\text { MRE }=\frac{\text { observed CD }(\mathrm{mdeg})}{C_{p} n l \times 10},
$$

where $C_{p}$ is the molar concentration of the protein, $n$ is the number of amino acid residues, and $l$ is the path length. The quantitative analysis of the $\alpha$-helix content of BSA was calculated from MRE values at $208 \mathrm{~nm}$ by the following equation [41]:

$$
\alpha \text {-helix }(\%)=\frac{-\mathrm{MRE}_{208}-4000}{33000-4000} \times 100 .
$$

As seen in Figure 7, the CD spectra of BSA exhibit two negative bands in UV region at 208 and $220 \mathrm{~nm}$, which are characteristic of $\alpha$-helix structure of protein [42]. The interaction between CA (or LB) and BSA causes a slight decrease in band intensity at all wavelengths of the UV region but without any significant shift in the peak position. The $\alpha$-helix content of BSA increased with the addition of CA or LB when the molar ratio of drug/BSA was 5:1 and 10:1. Furthermore, the increase of $\alpha$-helix of LB was slightly less than CA, which further confirms that the interaction between LB and BSA can be influenced by methoxyl group at 4substitution. From the previous results, it is evident that the effect of CA or LB on BSA caused secondary structure changes of the protein with the increase of helical stability.

\section{Conclusions}

In this work, the interaction between CA and BSA, LB, and BSA had been studied using fluorescence spectroscopy, 
ultraviolet spectroscopy, and circular dichroism at different temperatures under imitated physiological conditions. The results showed that the quenching mechanism of fluorescence of BSA by CA (or LB) was static quenching at low concentrations of CA (or LB), while a combined quenching (dynamic and static quenching) at higher concentrations of CA (or LB). Moreover, the quenching efficiency, binding affinity of LB to BSA, was more than that of CA and the donor to acceptor distance $r_{\mathrm{LB}}$ (1.73) is less than $r_{\mathrm{CA}}$ (2.01), which indicated that the 4-methoxyl group was more helpful to the ligands binding to proteins. The values of $n$ were approximately equal to 1 , so there was one binding site between CA and BSA, $\mathrm{LB}$, and BSA. The corresponding thermodynamic parameters were also obtained and indicated that electrostatic forces play a major role in the formation of the LB-BSA complex, but probably a combined force for CA-BSA complex. The results of synchronous fluorescence spectroscopy demonstrated that the polarity around tryptophan residues was increased and the hydrophobicity was decreased when CA (or LB) interacted with BSA, and CD spectra demonstrated that the secondary structures of BSA were changed to varying degrees by the binding of CA (or LB). This study is expected to provide some valuable data for the transportation and distribution of CA and LB in vivo and to be helpful to drug design and pharmaceutical research.

\section{Abbreviations}

CA: Cochinchinenin A

LB: Loureirin B

BSA: Bovine serum albumin

HAS: Human serum albumin

CD: Circular dichroism

Trp: Tryptophan.

\section{Acknowledgments}

This work was financially supported by the National Natural Science Foundation of China (nos. 21205145, 21276006, and 21036009), the Open Funds of State Key Laboratory of Chemo/Biosensing and Chemometrics of Hunan University (no. 201111), the Special Fund for Basic Scientific Research of Central Colleges, South-Central University for Nationalities (nos. CZZ10005, CZQ11012, and XTZ10001), the "FiveTwelfth" National Science and Technology Support Program (2012BAI27B00).

\section{References}

[1] D. Gupta, B. Bleakley, and R. K. Gupta, "Dragon's blood: botany, chemistry and therapeutic uses," Journal of Ethnopharmacology, vol. 115, no. 3, pp. 361-380, 2008.

[2] X. H. Wang, M. Gong, L. Tang et al., "Cloning, bioinformatics and the enzyme activity analyses of a phenylalanine ammonialyase gene involved in dragon's blood biosynthesis in Dracaena cambodiana," Molecular Biology Reports, vol. 40, no. 1, pp. 97$107,2012$.

[3] X. H. Wang, C. Zhang, L. L. Yang, and J. Gomes-Laranjo, "Production of dragon's blood in Dracaena cochinchinensis plants by inoculation of Fusarium proliferatum," Plant Science, vol. 180, no. 2, pp. 292-299, 2011.

[4] Y. D. Zhu, P. Zhang, H. P. Yu, J. Li, M. W. Wang, and W. M. Zhao J, "Anti-Helicobacter pylori and thrombin inhibitory components from Chinese dragon's blood, Dracaena cochinchinensis," Journal of Natural Products, vol. 70, no. 10, pp. 1570-1577, 2007.

[5] N. Xin, Y. J. Li, Y. Li et al., “Dragon's blood extract has antithrombotic properties, affecting platelet aggregation functions and anticoagulation activities," Journal of Ethnopharmacology, vol. 135, no. 2, pp. 510-514, 2011.

[6] T. Yi, H. B. Chen, Z. Z. Zhao, Z. L. Yu, and Z. H. Jiang, "Comparison of the chemical profiles and anti-platelet aggregation effects of two "dragon's blood" drugs used in traditional Chinese medicine," Journal of Ethnopharmacology, vol. 133, no. 27, pp. 796-802, 2011.

[7] Y. S. Li, J. X. Wang, M. M. Jia, M. Liu, X. J. Li, and H. B. Tang, "Dragon's blood inhibits chronic inflammatory and neuropathic pain responses by blocking the synthesis and release of substance P in rats," Journal of Pharmacological Sciences, vol. 118, no. 1, pp. 43-54, 2012.

[8] S. Chen, X. J. Huang, and X. M. Liu, "Reverse pharmacology and the R\&D of modern analgesic based on traditional medicines," Traditional Chinese Medicine, vol. 1, pp. 1-8, 2012.

[9] C. Dufour and O. Dangles, "Flavonoid-serum albumin complexation: determination of binding constants and binding sites by fluorescence spectroscopy," Biochimica et Biophysica Acta (BBA), vol. 1721, no. 1-3, pp. 164-173, 2005.

[10] E. H. Liu, L. W. Qi, and P. Li, "Structural relationship and binding mechanisms of five flavonoids with bovine serum albumin," Molecules, vol. 15, no. 12, pp. 9092-9103, 2010.

[11] J. Ghuman, P. A. Zunszain, I. Petitpas, A. A. Bhattacharya, M. Otagiri, and S. Curry, "Structural basis of the drug-binding specificity of human serum albumin," Journal of Molecular Biology, vol. 353, no. 1, pp. 38-52, 2005.

[12] Y. N. Ni, S. S. Wang, and S. Kokot, "Spectrometric study of the interaction between alpinetin and bovine serum albumin using chemometrics approaches," Analytica Chimica Acta, vol. 663, no. 2, pp. 139-146, 2010.

[13] D. C. Carter, X. M. He, P. D. Twigg et al., "Three-dimensional structure of human serum albumin," Science, vol. 244, no. 4909, pp. 1195-1198, 1989.

[14] Z. F. Yang, R. R. Tang, and Z. F. Zhang, "Synthesis and luminescent properties of $\mathrm{Tb}(\mathrm{III})$ complex with a novel pyrazolone ligand and its interaction with bovine serum albumin," Journal of Molecular Structure, vol. 1030, pp. 19-25, 2012.

[15] L. Trnková, I. Boušová, V. Staňková, and J. Dršata, "Study on the interaction of catechins with human serum albumin using spectroscopic and electrophoretic techniques," Journal of Molecular Structure, vol. 985, no. 2-3, pp. 243-250, 2011.

[16] H. M. Zhang, T. T. Chen, Q. H. Zhou, and Y. Q. Wang, "Binding of caffeine, theophylline, and theobromine with human serum albumin: a spectroscopic study," Journal of Molecular Structure, vol. 938, no. 1-3, pp. 221-228, 2009.

[17] Y. Z. Zhang, J. Zhang, F. F. Li, X. Xiang, A. Q. Ren, and Y. Liu, "Studies on the interaction between benzophenone and bovine serum albumin by spectroscopic methods," Molecular Biology Reports, vol. 38, no. 4, pp. 2445-2453, 2011.

[18] X. Y. Yu, R. H. Liu, F. X. Yang et al., "Study on the interaction between dihydromyricetin and bovine serum albumin by spectroscopic techniques," Journal of Molecular Structure, vol. 985, no. 2-3, pp. 407-412, 2011. 
[19] I. M. Vlasova, V. V. Zhuravleva, A. A. Vlasov, and A. M. Saletsky, "Interaction of cationic surfactant cethyltrimethylammonium bromide with bovine serum albumin in dependence on $\mathrm{pH}$ : a study of tryptophan fluorescence," Journal of Molecular Structure, vol. 1034, pp. 89-94, 2013.

[20] J. Zhang, X. J. Wang, Y. J. Yan, and W. S. Xiang, "Comparative studies on the interaction of genistein, 8-chlorogenistein, and $3^{\prime}, 8$-dichlorogenistein with bovine serum albumin," Journal of Agricultural and Food Chemistry, vol. 59, no. 13, pp. 7506-7513, 2011.

[21] T. Peter, "Serum albumin," Advances in Protein Chemistry, vol. 37, pp. 161-245, 1985.

[22] D. Romanini, G. Avalle, B. Farruggia, B. Nerli, and G. Picó, "Spectroscopy features of the binding of polyene antibiotics to human serum albumin," Chemico-Biological Interactions, vol. 115, no. 3, pp. 247-260, 1998.

[23] X. Y. Yu, Q. Yao, W. Li et al., "Investigation of the interaction between $N$-benzyl piperidones and bovine serum albumin by spectroscopic approaches," Journal of Solution Chemistry, vol. 41, no. 10, pp. 1747-1758, 2012.

[24] J. Q. Tong, H. X. Zhang, H. M. Yang, and P. Mei, "Photochemical studies on the binding of an organic fluoride to bovine serum albumin," Molecular Biology Reports, vol. 37, no. 4, pp. 1741-1747, 2010.

[25] J. R. Lakowicz, Principles of Fluorescence Spectroscopy, Plenum Press, New York, NY, USA, 2nd edition, 1999.

[26] J. R. Lakowicz and G. Weber, "Quenching of fluorescence by oxygen. A probe for structural fluctuations in macromolecules," Biochemistry, vol. 12, no. 21, pp. 4161-4170, 1973.

[27] Z. H. Hou, N. B. Zhou, B. H. He, Y. Yang, and X. Y. Yu, "Study of the interaction between quinocetone and bovine serum albumin by spectroscopic approaches," Journal of Molecular Structure, vol. 1004, no. 1-3, pp. 116-120, 2011.

[28] S. Y. Lu, X. Y. Yu, Y. Yang, and X. F. Li, "Spectroscopic investigation on the intermolecular interaction between $\mathrm{N}$-confused porphyrins-(3-methylisoxazole) diad and bovine serum albumin," Spectrochimica Acta A, vol. 99, pp. 116-121, 2012.

[29] Y. N. Ni, F. Y. Zhang, and S. Kokot, "Enantioselective binding interaction of the metolachlor pesticide enatiomers with bovine serum albumin-a spectroscopic analysis study," Spectrochimica Acta A, vol. 97, pp. 753-761, 2012.

[30] D. M. Chipman, V. Grisaro, and N. Sharon, "The binding of oligosaccharides containing $\mathrm{N}$-acetylglucosamine and $\mathrm{N}$ acetylmuramic acid to lysozyme. The specificity of binding subsites," The Journal of Biological Chemistry, vol. 242, no. 19, pp. 4388-4394, 1967.

[31] T. H. Wang, Z. M. Zhao, B. Z. Wei, L. Zhang, and L. Ji, "Spectroscopic investigations on the binding of dibazol to bovine serum albumin," Journal of Molecular Structure, vol. 970, no. 1-3, pp. 128-133, 2010.

[32] P. D. Ross and S. Subramanian, "Thermodynamics of protein association reactions: forces contributing to stability," Biochemistry, vol. 20, no. 11, pp. 3096-3102, 1981.

[33] M. Ehteshami, F. Rasoulzadeh, S. Mahboob, and M. R. Rashidi, "Characterization of 6-mercaptopurine binding to bovine serum albumin and its displacement from the binding sites by quercetin and rutin," Journal of Luminescence, vol. 135, pp. 164169, 2013.

[34] J. Jayabharathi, V. Thanikachalam, R. Sathishkumar, and K. Jayamoorthy, "Fluorescence investigation of the interaction of 2-(4-fluorophenyl)-1-phenyl-1H-phenanthro [9,10-d] imidazole with bovine serum albumin," Journal of Photochemistry and Photobiology B, vol. 117, pp. 222-227, 2012.

[35] T. Förster and O. Sinanoglu, Eds., Modern Quantum Chemistry, vol. 3, Academic Press, New York, NY, USA, 1966.

[36] Y. Liu, M. M. Chen, and L. Song, "Comparing the effects of $\mathrm{Fe}(\mathrm{III})$ and $\mathrm{Cu}(\mathrm{II})$ on the binding affinity of erlotinib to bovine serum albumin using spectroscopic methods," Journal of Luminescence, vol. 134, pp. 515-523, 2013.

[37] Y. Z. Zhang, J. Dai, X. P. Zhang, X. Yang, and Y. Liu, "Studies of the interaction between Sudan I and bovine serum albumin by spectroscopic methods," Journal of Molecular Structure, vol. 888, no. 1-3, pp. 152-159, 2008.

[38] A. Varlan and M. Hillebrand, "Bovine and human serum albumin interactions with 3-carboxyphenoxathiin studied by fluorescence and circular dichroism spectroscopy," Molecules, vol. 15, no. 6, pp. 3905-3919, 2010.

[39] J. N. Miller, "Recent developments in fluorescence and chemiluminescence analysis: plenary lecture," The Analyst, vol. 109, no. 3, pp. 191-198, 1984.

[40] R. W. Gongdon, G. W. Muth, and A. G. Splittgerber, "The binding interaction of coomassie blue with proteins," Analytical Biochemistry, vol. 213, no. 2, pp. 407-413, 1993.

[41] X. J. Guo, X. W. Han, J. Tong et al., "The investigation of the interaction between piracetam and bovine serum albumin by spectroscopic methods," Journal of Molecular Structure, vol. 966, no. 1-3, pp. 129-135, 2010.

[42] F. Ding, W. Liu, N. Li, L. Zhang, and Y. Sun, "Complex of nicosulfuron with human serum albumin: a biophysical study," Journal of Molecular Structure, vol. 975, no. 1-3, pp. 256-264, 2010. 

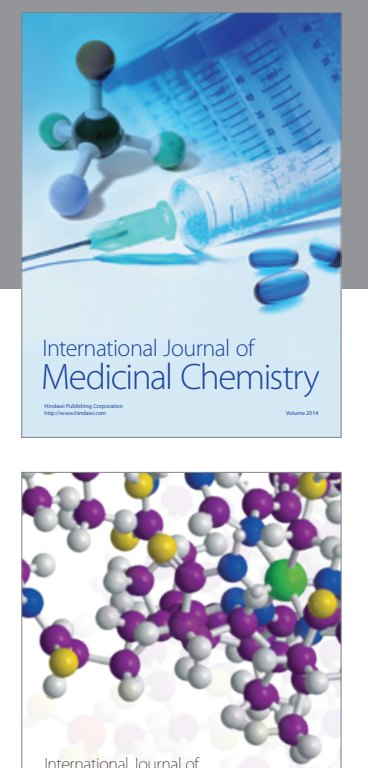

\section{Carbohydrate} Chemistry

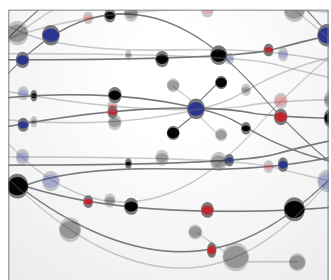

The Scientific World Journal
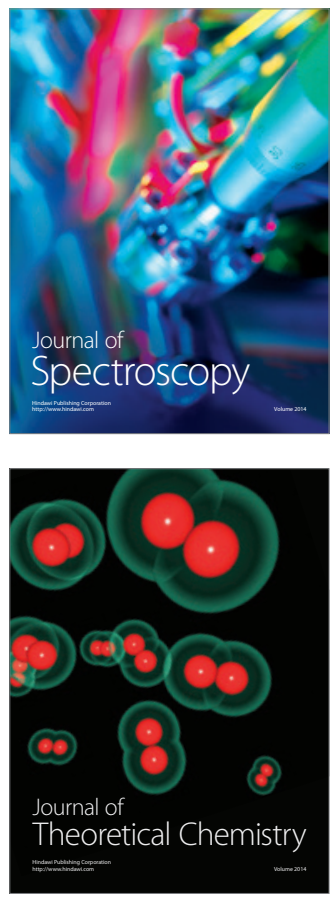
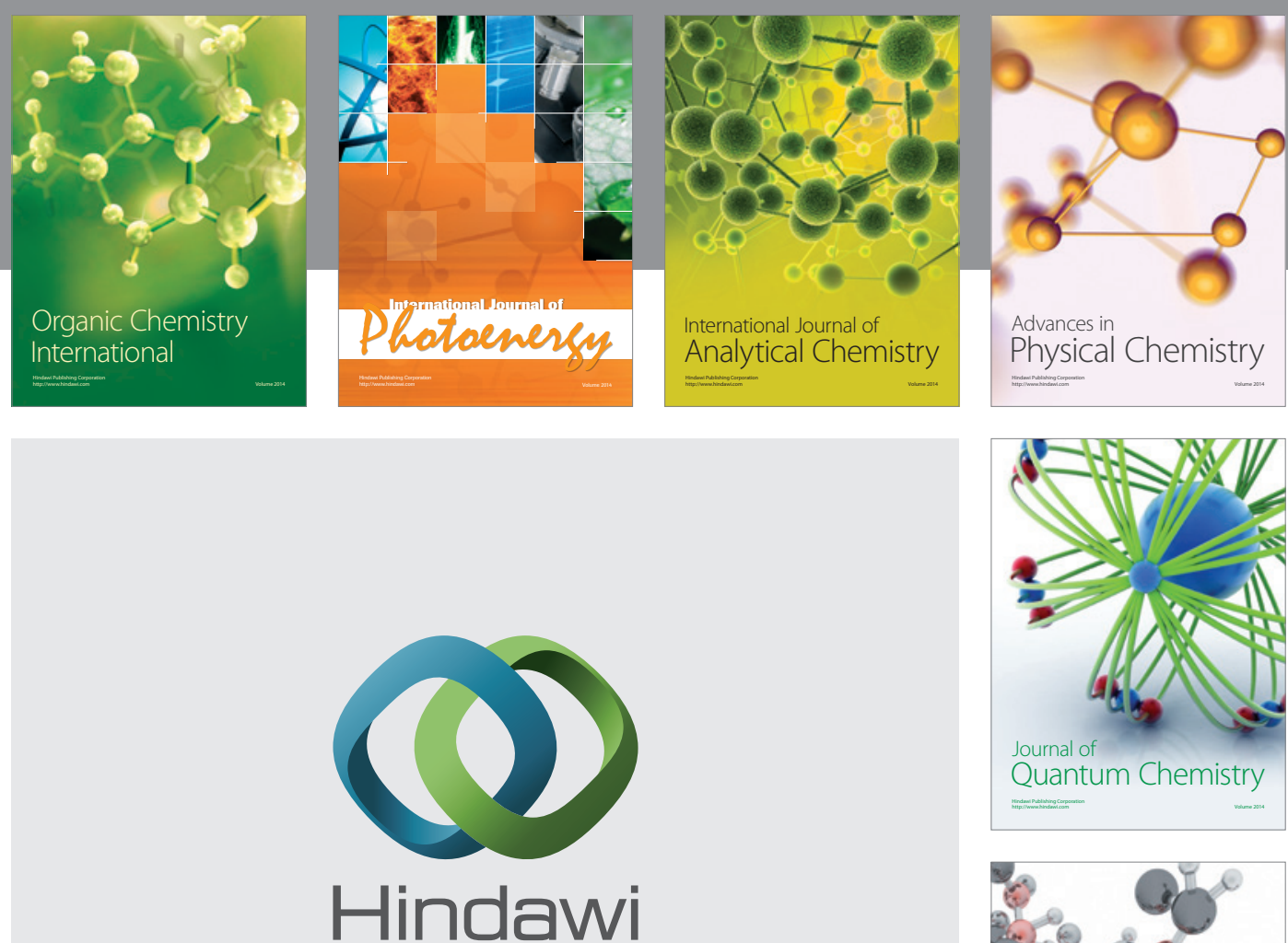

Submit your manuscripts at

http://www.hindawi.com

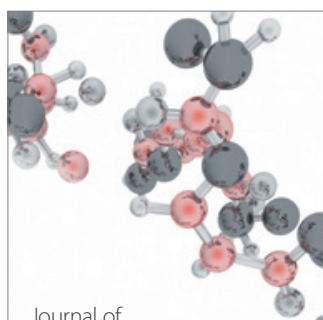

Analytical Methods

in Chemistry

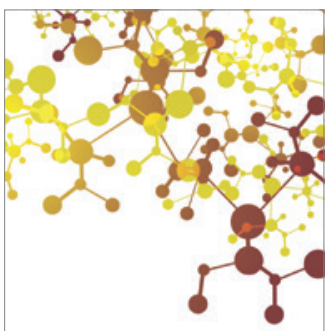

Journal of

Applied Chemistry

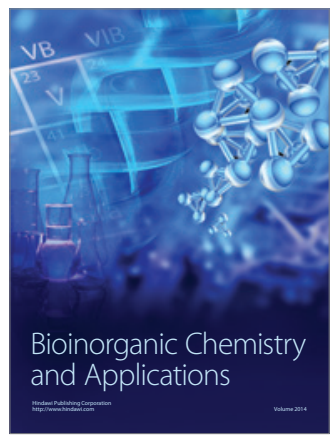

Inorganic Chemistry
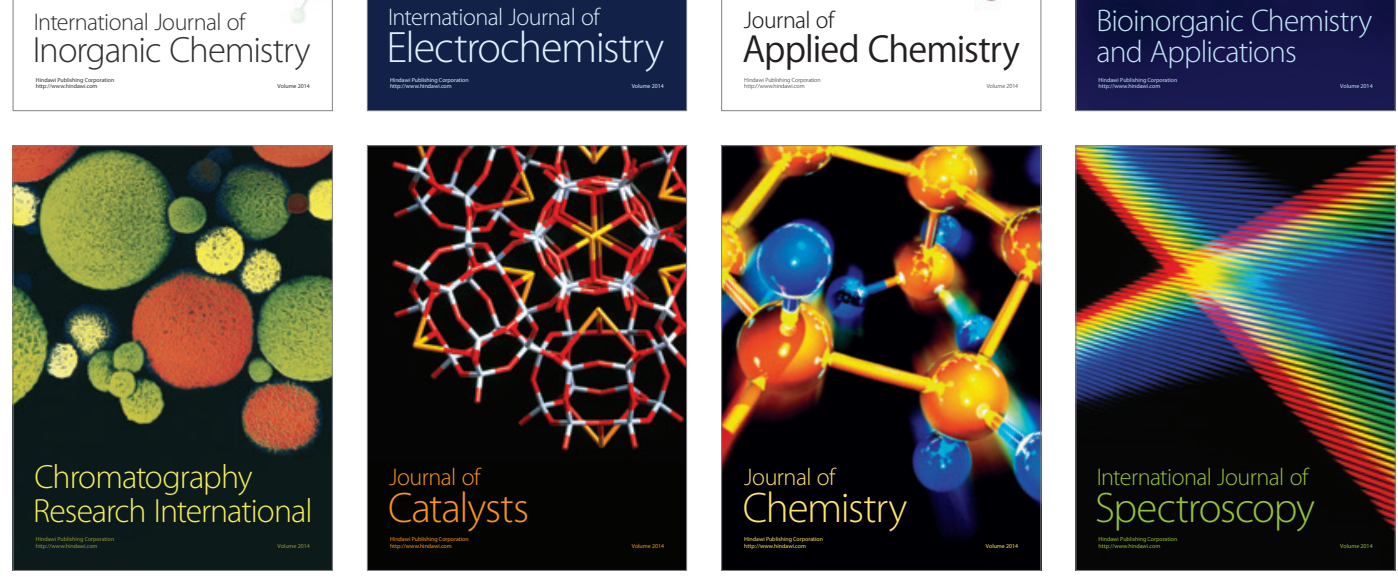